\title{
A Simple Method to Synthesize Lignin Nanoparticles
}

\author{
Pawan Kumar Mishra ${ }^{1, *(\mathbb{D})}$ and Adam Ekielski ${ }^{2}$ (D) \\ 1 Department of Wood Processing, Mendel University in Brno, 61300 Brno, Czech Republic \\ 2 Department of Production Management and Engineering, Warsaw University of Life Sciences, \\ 02-787 Warsaw, Poland; adam_ekielski@sggw.pl \\ * Correspondence: xmishra@mendelu.cz
}

Received: 15 April 2019; Accepted: 8 June 2019; Published: 10 June 2019

\begin{abstract}
The self-assembly of lignin (molecular and supramolecular) is driven mainly by non-covalent interactions, and the nature of the solvents and antisolvents directly affect the driving forces. The lignin particle is usually formed by noncovalently bonded cylindrical subunits. In this paper, we report a simple method which can be used to synthesize lignin nanoparticles by using spray freezing. The method is based on two properties of dimethyl sulfoxide (DMSO) that are excellent lignin solubility and a high melting point. Based on these two properties, kraft lignin solution in DMSO was sprayed onto liquid nitrogen-cooled copper plates using a handheld spray. The high melting point of DMSO caused immediate freezing and particle formation. The obtained particles were characterized for their size and morphology using dynamic light scattering (DLS), as well as scanning electron microscopy (SEM). Nano-range polydispersed particles were obtained by spraying $0.05 \%$ of lignin onto DMSO. This method can avoid lignin-solvent-antisolvent interactions, and can also be used to study lignin-lignin (subunits) and lignin-DMSO interactions.
\end{abstract}

Keywords: Self -assembly; Lignin nanoparticles; Spray-freezing

\section{Introduction}

Nanomaterials and nanotechnology help to create new strategies for biomass valorization, as well as its applications [1-5]. Lignin is an aromatic polymer, commercially produced as a byproduct from the paper mills. It is mostly burnt for energy purposes. Various approaches have been developed to try and valorize technical lignin (obtained as a byproduct of different industrial pulping processes), and even lignin centric biorefineries have also been suggested [6]. Chemically, lignin monomers can be generalized as guaiacol, syringol, and conferiyl alcohol. The relative composition and bonding system of lignin varies from source to source. A conventional system of numbering used in lignin nomenclature is shown in Figure 1. For nanoparticle formation involving the self-assembly of smaller subunits of lignin by non-covalent interactions, the structure of lignin is highly important. The structure of lignin further varies with the source, extraction process, and extent of condensation. Therefore, any valorization strategy must include either extensive characterization, or a source that is globally available. Lignin nanoparticles can play an important role in high-value utilization of technical lignin; therefore, several methods have been proposed for lignin nanoparticle synthesis [7-10]. Most of the methods involve the self-assembly of lignin, which, in turn, is affected by the nature or type of the solvent, type of noncovalent interaction, the structure of technical lignin, the extent of condensation, and also by solvent-lignin-antisolvent interactions [11,12]. Therefore, different approaches for the synthesis of lignin nanoparticles also provide an excellent platform for the study of inter- and intramolecular interactions of technical lignins, their solution structure, and their solvent-solute interactions $[13,14]$. Several reviews on lignin nanoparticle synthesis and its underlying processes have already addressed the issue in detail $[7,11,13]$. 


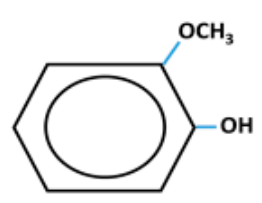

Guaiacol
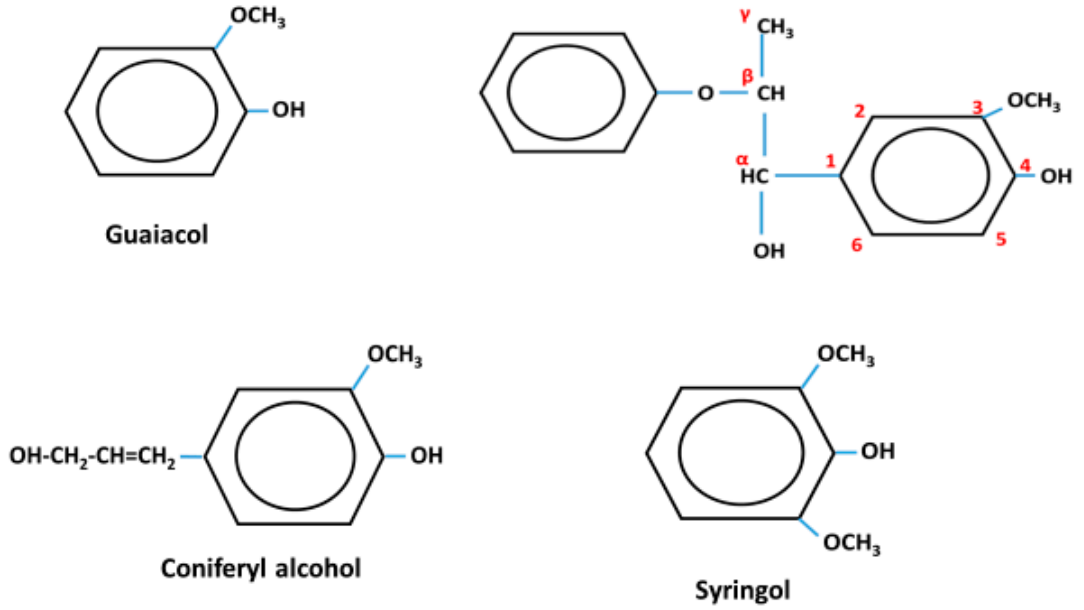

Figure 1. The monomers and numbering system used in lignin.

Lignin nanoparticles can have applications in agriculture, biocomposites, biomedical science, coatings, and many other fields [6,7]. The number of approaches used for lignin nanoparticles from a solution either involve precipitation from a solvent using some sort of antisolvent (involving solvent-solute-antisolvent interactions), or atomization and droplet processing (using the one drop, one particle approach) [13]. In our previous study, we reported a method using an ultrasonic nebulizer [15], which needed complex assembly and instrumentation. In this study, we suggest a simple method that can be used in any lab without any special equipment for lignin nanoparticle synthesis. Additionally, the simplicity of the method will help the upscaling process of the suggested method into spray-freeing onto a cryogenic liquid. As the method only involves one solvent during particle formation, it can also easily be used to study solvent-lignin interactions.

\section{Materials and Methods}

\subsection{Materials}

The materials used in this study included alkali lignin (Sigma Aldrich), syringe filters (AXIVA - Nylon Syringe Filters-Sterile, Axiva Scichem Biotech Pvt, USA), and a dialysis bag (D9777, dialysis tubing cellulose membrane, $14 \mathrm{kD}$ mol. Wt., Sigma-Aldrich ${ }^{\circledR}$, USA).

\subsection{Synthesis of Lignin Nanoparticles}

Lignin was dissolved $(0.05 \%)$ in DMSO under constant magnetic stirring for $1 \mathrm{~h}$. The solution was then filtered through a $0.45 \mu \mathrm{m}$ syringe filter to remove undissolved material. The solution thus obtained was sprayed onto a liquid $\mathrm{N}_{2}$ cooled copper plate (Figure 2). As the droplets strike against the $\mathrm{Cu}$ plate, they become lignin-entrapped frozen droplets. The frozen mass was collected from a copper plate, suspended in water at $4{ }^{\circ} \mathrm{C}$, and sonicated for $5 \mathrm{~min}$. This suspension was then lyophilized to obtain a dry powder, and this powder was suspended in deionized water and then sonicated for $3 \mathrm{~min}$. This suspension was used for further study.

\subsection{Particle Size Determination}

The lyophilized particles were suspended in deionized water and sonicated for $2 \mathrm{~min}$. The suspension thereby obtained was measured for particle size using a Zetasizer nano ZS (Malvern, PA, USA).

\subsection{Scanning Electron Microscopy (SEM)}

The lyophilized particles were suspended in deionized water and sonicated for $2 \mathrm{~min}$. A drop of this suspension was placed onto a sample holder and dried at room temperature. After drying, 
the sample was sputter-coated (ABG7340, Agar sputter coater, Agar scientific, UK) by a layer of gold using argon as a carrier gas at a pressure of 0.5 bars. The samples were observed using SEM (EVO 18, Zeiss, Germany).

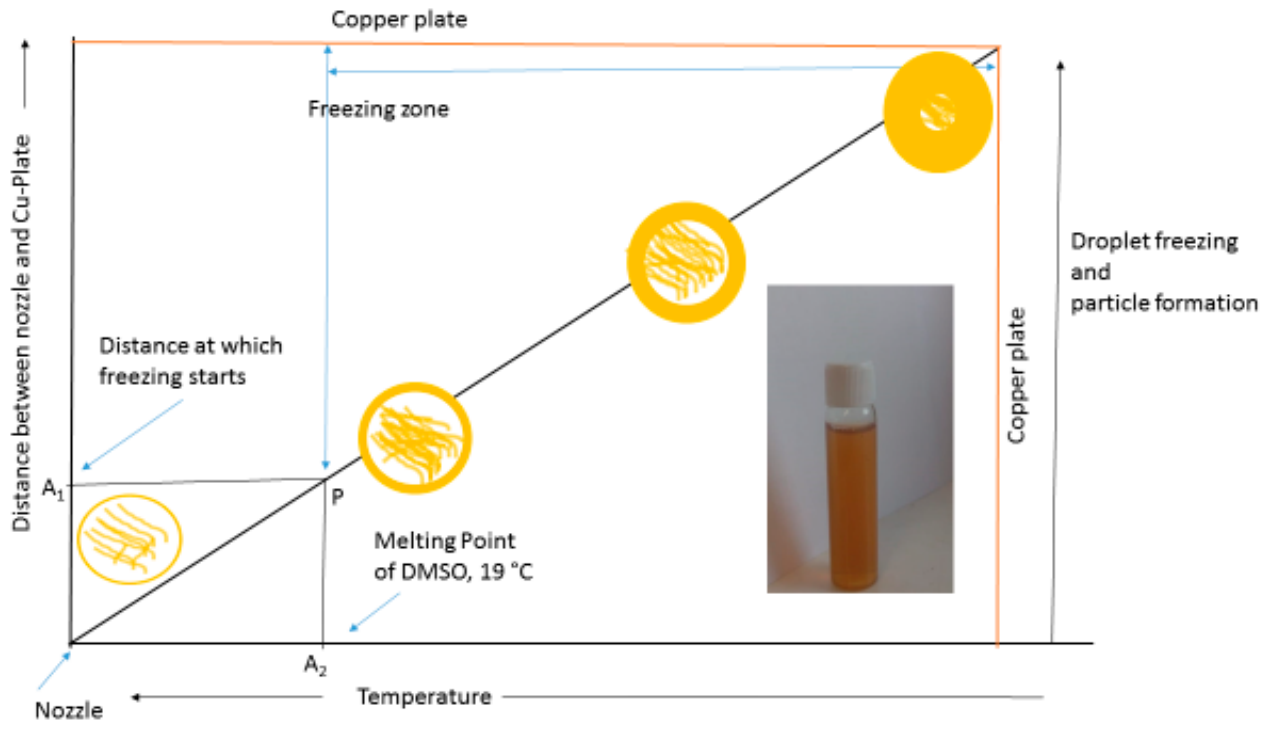

Figure 2. Graphical presentation of the method used and the mechanism of particle formation.

\subsection{Transmission Electron Microscopy (TEM)}

The samples for TEM imaging were prepared by drying a droplet of diluted particle suspension onto copper grids coated with a thin carbon film for $24 \mathrm{~h}$ at room temperature. Images were obtained using a FEI technai F30 electron microscope (Oregon, USA) with an accelerating voltage of $300 \mathrm{kV}$.

\section{Results and Discussion}

As presented in Figure 2, as the droplet leaves the nozzle and moves toward the copper plate (pre-cooled using liquid nitrogen), two parameters change simultaneously: firstly, the distance between the copper plate and droplet that decreases with time (represented on the Y-axis), and secondly, the temperature of the drop (represented on the $\mathrm{X}$-axis). As the temperature of the droplet falls below the melting point of DMSO, the freezing process begins (represented by $\mathrm{A}_{1}, \mathrm{~A}_{2,}$, and $\mathrm{P}$ on different axes); this zone with a temperature below the freezing point of DMSO can also be called a freezing zone. At this time, the outside-in or inside-out freezing of the particle decides its morphology [15]. The outside-in freezing results into solid particle formation, while inside-out freezing leads to a hollow particle formation. We observed and reported this phenomenon in the case of lignin in our previous study [15]. The size of the particles can be easily calculated from the size of the droplet by applying the one drop, one particle hypothesis, as well as the conservation of mass principle; however, this study aimed to report a simple and easily scalable method for lignin nanoparticle synthesis. The role of the solvent is very specific in the synthesis of the lignin nanoparticle. The solvent-solute (lignin) noncovalent interactions involved in solubilizing the lignin vary with the choice of solvent. Yang et al. [14] suggested the absence of hydrogen bonds in the dissolution of kraft lignin in DMSO; however, ethylene glycol (EG) required the contribution of hydrogen bonding for kraft lignin dissolution. It is also commonly accepted that the lignin nanoparticle is composed of cylindrical subunits bound together by noncovalent interactions $[14,16]$. On the basis of the above two studies, the role of hydrogen bonding in lignin nanoparticle formation from a DMSO solution can be assumed to be none or minimal.

In the process of spray-freezing, the mechanism of particle formation can be explained by the one drop, one particle hypothesis. When being sprayed onto the pre-cooled copper plate (using liquid 
nitrogen), the drop travels toward the plate and the temperature of the droplet falls. As it reaches the melting point of DMSO, the droplet starts to freeze from the outside to the inside, leading to particle formation (Figure 2). The main forces involved in particle formation can be assumed to be the $\pi-\pi$ interaction/stacking, as well as the Van der Waal forces. In the case of lignin, the flat, disc-shaped configuration of the lignin molecule favors face-centered configuration in stacking [17]. The studies on sodium lignosulphonate suggested the formation of J-type aggregate in lignin during $\pi-\pi$ stacking. This information is not available for alkali lignin. The role of hydrogen bonding and hydrophobic interactions can be ignored due to the presence of only DMSO at the time of particle formation and the absence of the aqueous phase, respectively $[14,18]$.

The hydrophobic interaction has been commonly used as a contributory noncovalent force, especially in cases that involve an aqueous antisolvent $[19,20]$. The dynamic light scattering (DLS) data showed a particle size of $150 \mathrm{~nm}$ with a PDI (polydispersity index) value of 0.5 . The high PDI value can be expected by the variation in droplet size from a hand-held spray bottle. However, the obtained particles were well into the nanometer range. The SEM images (Figure 3) show a clear fractal-like the image at a lower resolution; however, spherical particles can be seen in the other three images. The spherical particles were further confirmed by TEM images (Figure 4). The fractal-like structure could also be due to nanoscale covalent interactions that were otherwise screened by the presence of a solvent. However, the fractal-like structure needs further investigations on the solvent evaporation dynamics and particle-solvent interaction before arriving at any conclusions.

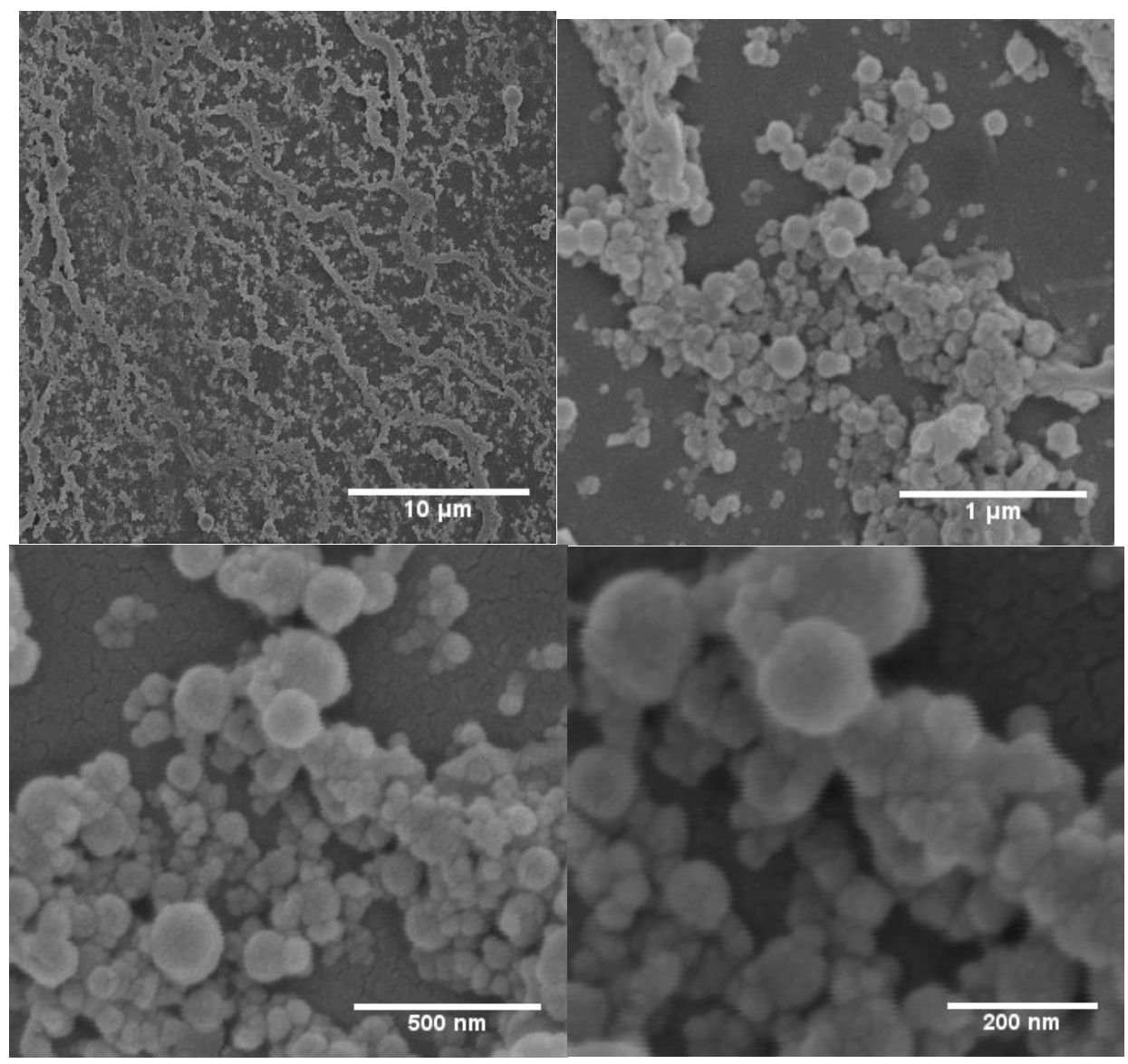

Figure 3. Particles prepared by spray-freezing, then dried at room temperature and neutral $\mathrm{pH}$. 


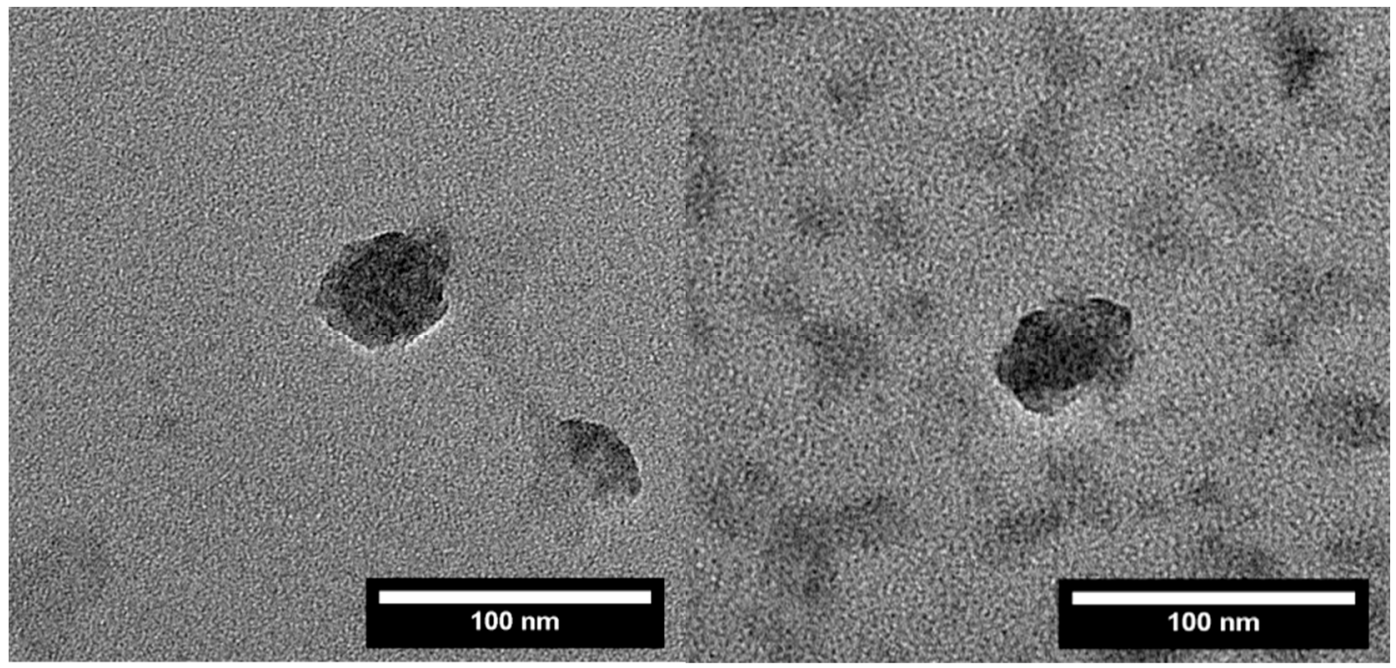

Figure 4. Transmission electron microscopy (TEM) images of representative lignin nanoparticles.

\section{Conclusions}

From this paper, it was shown that a simple lab scale set-up can yield lignin spherical lignin nanoparticles. These particles were self-assembled using a one drop, one particle approach. As the lignin nanoparticle is composed of cylindrical subunits of a few nanometers that form the spherical particle, the suggested approach can be used to study lignin-lignin (subunits) and lignin-DMSO interactions that are mostly noncovalent. Additionally, the scalability of the process can be used to develop spray-freeing onto cryogenic-type reactors for lignin valorization. The excellent solubility of technical lignins in DMSO is useful when valorizing technical lignins.

Author Contributions: Conceptualization and Methodology, P.K.M. and A.E.; Writing reviewing and editing P.K.M.

Funding: This research received no external funding.

Conflicts of Interest: The authors declare no conflict of interest.

\section{References}

1. Siddiqui, L.; Mishra, H.; Mishra, P.K.; Iqbal, Z.; Talegaonkar, S. Novel 4-in-1 strategy to combat colon cancer, drug resistance and cancer relapse utilizing functionalized bioinspiring lignin nanoparticle. Med. Hypotheses 2018, 121, 10-14. [CrossRef] [PubMed]

2. Král, P.; Klímek, P.; Mishra, P.K.; Rademacher, P.; Wimmer, R. Preparation and Characterization of Cork Layered Composite Plywood Boards. BioResources 2014, 9, 1977-1985. [CrossRef]

3. Mishra, P.K.; Giagli, K.; Tsalagkas, D.; Mishra, H.; Talegaonkar, S.; Gryc, V.; Wimmer, R. Changing Face of Wood Science in Modern Era: Contribution of Nanotechnology. Recent Pat. Nanotechnol. 2018, 12, 13-21. [CrossRef] [PubMed]

4. Mittal, N.; Jansson, R.; Widhe, M.; Benselfelt, T.; Håkansson, K.M.O.; Lundell, F.; Hedhammar, M.; Söderberg, L.D. Ultrastrong and Bioactive Nanostructured Bio-Based Composites. ACS Nano 2017, 11, 5148-5159. [CrossRef] [PubMed]

5. Mittal, N.; Ansari, F.; Gowda, V.K.; Brouzet, C.; Chen, P.; Larsson, P.T.; Roth, S.V.; Lundell, F.; Wågberg, L.; Kotov, N.A.; et al. Multiscale Control of Nanocellulose Assembly: Transferring Remarkable Nanoscale Fibril Mechanics to Macroscale Fibers. ACS Nano 2018, 12, 6378-6388. [CrossRef] [PubMed]

6. Constant, S.; Wienk, H.L.; Frissen, A.E.; de Peinder, P.; Boelens, R.; Van Es, D.S.; Grisel, R.J.; Weckhuysen, B.M.; Huijgen, W.J.; Gosselink, R.J. New insights into the structure and composition of technical lignins: A comparative characterisation study. Green Chem. 2016, 18, 2651-2665. [CrossRef] 
7. Figueiredo, P.; Lintinen, K.; Hirvonen, J.T.; Kostiainen, M.A.; Santos, H.A. Properties and chemical modifications of lignin: Towards lignin-based nanomaterials for biomedical applications. Prog. Mater. Sci. 2018, 93, 233-269. [CrossRef]

8. Frangville, C.; Rutkevicius, M.; Richter, A.P.; Velev, O.D.; Stoyanov, S.D.; Paunov, V.N. Fabrication of Environmentally Biodegradable Lignin Nanoparticles. Chemphyschem 2012, 13, 4235-4243. [CrossRef] [PubMed]

9. Duval, A.; Lawoko, M. A review on lignin-based polymeric, micro- and nano-structured materials. React. Funct. Polym. 2014, 85, 78-96. [CrossRef]

10. ur Rahman, O.; Shi, S.; Ding, J.; Wang, D.; Ahmad, S.; Yu, H. Lignin nanoparticles: synthesis, characterization and corrosion protection performance. New J. Chem. 2018, 42, 3415-3425. [CrossRef]

11. Beisl, S.; Miltner, A.; Friedl, A. Lignin from Micro-to Nanosize: Production Methods. Int. J. Mol. Sci. 2017, 18, 1244. [CrossRef] [PubMed]

12. Ago, M.; Tardy, B.L.; Wang, L.; Guo, J.; Khakalo, A.; Rojas, O.J. Supramolecular assemblies of lignin into nano-and microparticles. MRS Bull. 2017, 42, 371-378. [CrossRef]

13. Mishra, P.K.; Ekielski, A. The Self-Assembly of Lignin and Its Application in Nanoparticle Synthesis: A Short Review. Nanomaterials 2019, 9, 243. [CrossRef]

14. Yang, M.; Zhao, W.; Singh, S.; Simmons, B.; Cheng, G. On the solution structure of kraft lignin in ethylene glycol and its implication for nanoparticle preparation. Nanoscale Adv. 2018. [CrossRef]

15. Mishra, P.K.; Wimmer, R. Aerosol assisted self-assembly as a route to synthesize solid and hollow spherical lignin colloids and its utilization in layer by layer deposition. Ultrason. Sonochem. 2017, 35, 45-50. [CrossRef] [PubMed]

16. Salentinig, S.; Schubert, M. Softwood Lignin Self-Assembly for Nanomaterial Design. Biomacromolecules 2017. [CrossRef] [PubMed]

17. Deng, Y.; Feng, X.; Yang, D.; Yi, C.; Qiu, X. Pi-Pi STACKING OF THE AROMATIC GROUPS IN LIGNOSULFONATES. BioResources 2012, 7, 1145-1156.

18. Xiong, F.; Han, Y.; Wang, S.; Li, G.; Qin, T.; Chen, Y.; Chu, F. Preparation and Formation Mechanism of Renewable Lignin Hollow Nanospheres with a Single Hole by Self-Assembly. ACS Sustain. Chem. Eng. 2017, 5, 2273-2281. [CrossRef]

19. Chen, F.; Zhou, W.; Yao, H.; Fan, P.; Yang, J.; Fei, Z.; Zhong, M. Self-assembly of NiO nanoparticles in lignin-derived mesoporous carbons for supercapacitor applications. Green Chem. 2013, 15, 3057-3063. [CrossRef]

20. Lievonen, M.; Valle-Delgado, J.J.; Mattinen, M.-L.; Hult, E.-L.; Lintinen, K.; Kostiainen, M.A.; Paananen, A.; Szilvay, G.R.; Setälä, H.; Österberg, M. A simple process for lignin nanoparticle preparation. Green Chem. 2016, 18, 1416-1422. [CrossRef]

(C) 2019 by the authors. Licensee MDPI, Basel, Switzerland. This article is an open access article distributed under the terms and conditions of the Creative Commons Attribution (CC BY) license (http://creativecommons.org/licenses/by/4.0/). 